\title{
A realistic MR compatible aortic phantom to validate hemodynamic parameters from MRI data: aortic coarctation patients comparison using catheterization
}

\author{
Jesus Urbina ${ }^{1,4^{*}}$, Julio Sotelo ${ }^{1,5}$, Cristian Tejos ${ }^{1,5}$, Pablo Irarrazaval ${ }^{1,5}$, Marcelo E Andia ${ }^{1,6}$, Reza Razavi², \\ Israel Valverde ${ }^{3,7}$, Sergio Uribe ${ }^{1,6}$
}

From 18th Annual SCMR Scientific Sessions

Nice, France. 4-7 February 2015

\section{Background}

Recently, 3D printing technologies have emerged as a very innovative technique to produce anatomical replicas. Nevertheless, vessel phantoms built up to now are simplified models, with difficulties to obtain parameters with physiological values. The aim of this work is to show and validate a MR compatible thoracic aorta system, designed to obtain hemodynamic parameters within a range comparable to healthy volunteers and patients with aortic coarctation (AoCo).

\section{Methods}

The phantom is a closed circuit integrated by a MR compatible unit pump with a control unit (Shelley Medical Imaging Technologies) and a realistic aortic model built with flexible silicone (Elastrat). Three non-return valves were installed to avoid negative pressures. Additionally, shut-off valves were added to regulate the flow distribution between the different vessels. We built an $11 \mathrm{~mm}$ AoCo, which was placed in the descending aorta just after the left subclavian artery. Additionally, we equipped the system with a catheterization unit to measure invasive pressure along the AoCo.

Experiments were performed on a $1.5 \mathrm{~T}$ MR-system (Philips). Two different situations were studies: a phantom without and with an $11 \mathrm{~mm}$ AoCo. For each situation we acquired 2D and 3D PC-MRI data. The 2D PCMRI data were acquired in 5 section of the aorta (figure

'Biomedical Imaging Center, Pontificia Universidad Católica de Chile, Santiago, Chile

Full list of author information is available at the end of the article
1) and the 3D PC-MRI data were acquired in the entire phantom. Velocity and flow related parameters were obtained from both methods using the commercial software GTFlow 2.0.10 (Gyrotools LCC). We measured the systolic pressure gradient with the catheterization system.

Phantom data were compared with 3D PC-MRI data acquired in 10 healthy volunteers, and 2 unrepaired aortic coarctation patients. Patient data were acquired in a combined MRI/Catheter interventional suite.

\section{Results}

We obtained realistic hemodynamic parameters, similar to volunteers and patients values. Using 4D flow data in the position I (Ascending aorta), the aortic phantom without AoCo had a peak flow and velocity of $270 \mathrm{ml} / \mathrm{s}$ and $91 \mathrm{~cm} / \mathrm{s}$ and volunteers of $357 \mathrm{ml} / \mathrm{s}$ and $107 \mathrm{~cm} / \mathrm{s}$ respectively. Phantom with AoCo had a peak flow and velocity of $265 \mathrm{ml} / \mathrm{s}$ and $95 \mathrm{~cm} / \mathrm{s}$, patients 1 of $244 \mathrm{ml} / \mathrm{s}$ and $196 \mathrm{~cm} / \mathrm{s}$ and patient 2 of $376 \mathrm{ml} / \mathrm{s}$ and $187 \mathrm{~cm} / \mathrm{s}$ respectively (Figure 1). The $\mathrm{CO}$ in the phantom without AoCo and volunteers were 3.5 and $4.5 \mathrm{~L} / \mathrm{min}$. The CO in the phantom with AoCo and patients 1 and 2 were 3.0, 3.9 and $5.7 \mathrm{~L} / \mathrm{min}$. Phantom pressure without and with the AoCo were 131/58 and 127/51 $\mathrm{mmHg}$ with a trans-coarctation systolic pressure gradient of 10 mmHg. Patients 1 had a pressure of $120 / 51 \mathrm{mmHg}$ with a gradient of $20 \mathrm{mmHg}$. Patient 2 had a pressure of 87/ $39 \mathrm{mmHg}$ with a gradient of $11 \mathrm{mmHg}$ (Figure 2). Flow, velocity and pressure waveforms in the phantom without and with AoCo were similar to healthy volunteers and patients respectively. 


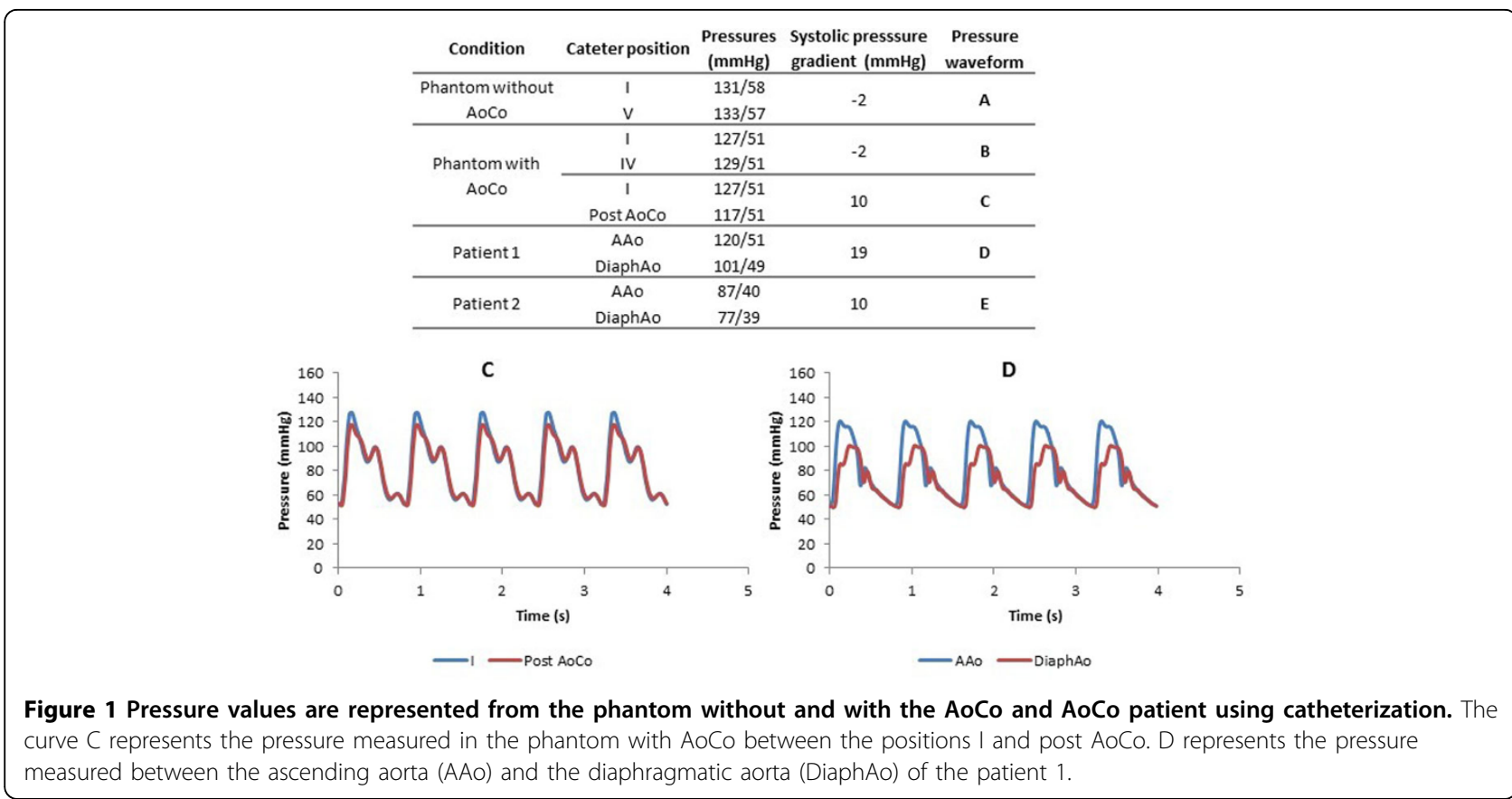

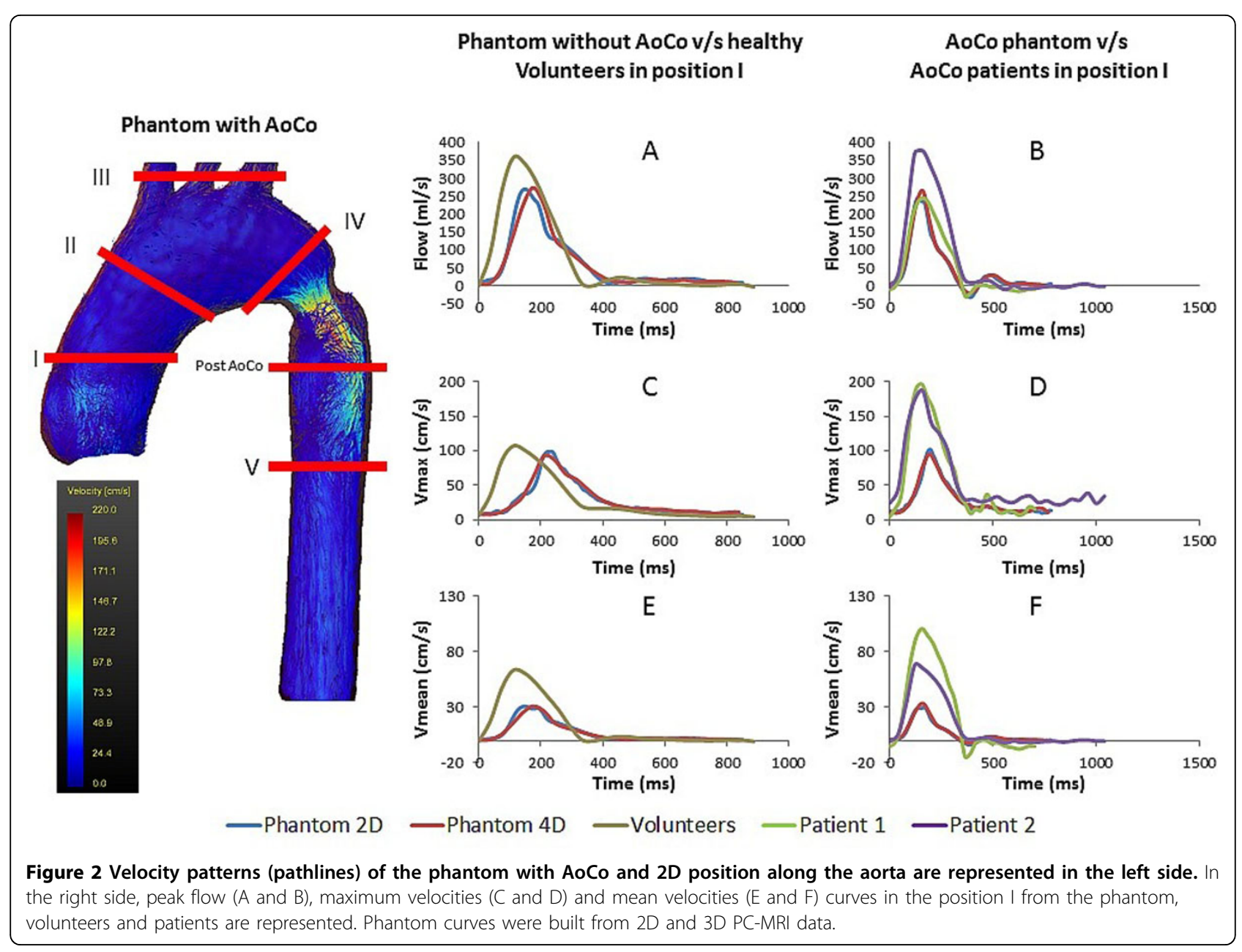




\section{Conclusions}

Results in this study demonstrate the feasibility of our realistic aortic system to simulate physiologic and pathologic hemodynamic parameters through an AoCo.

\section{Funding}

Grant Sponsor

Fondo Nacional de Desarrollo Científico y Tecnológico (FONDECYT), Ministerio de Educación, Chile. Grant Number: FONDECYT \#1141036.

\section{Authors' details}

'Biomedical Imaging Center, Pontificia Universidad Católica de Chile, Santiago, Chile. ${ }^{2}$ maging Sciences and Biomedical Engineering, King's College London, London, UK. ${ }^{3}$ Institute of Biomedicine of Seville, Universidad de Sevilla, Seville, Spain. ${ }^{4}$ School of Medicine, Pontificia Universidad Católica de Chile, Santiago, Chile. ${ }^{5}$ Electrical Engineering Department, Pontificia Universidad Católica de Chile, Santiago, Chile. ${ }^{6}$ Radiology Department, Pontificia Universidad Católica de Chile, Santiago, Chile. ${ }^{7}$ Cardiology Unit, Hospital Virgen del Rocío, Universidad de Sevilla, Seville, Spain.

Published: 3 February 2015

- Convenient online submission

- Thorough peer review

- No space constraints or color figure charges

- Immediate publication on acceptance

- Inclusion in PubMed, CAS, Scopus and Google Scholar

- Research which is freely available for redistribution 